\title{
Comparative Analysis of FBMC and OFDM Multicarrier Techniques for Wireless Communication Networks
}

\author{
Bidyalaxmi Devi \\ Tensubam \\ Post Graduate Scholar \\ DIT University, Dehradun
}

\author{
Nongmaithem Lalleima \\ Chanu \\ Post Graduate Scholar \\ DIT University, Dehradun
}

\author{
Sonika Singh, Ph.D \\ Associate Professor \\ DIT University, Dehradun
}

\begin{abstract}
Multicarrier (MC) techniques are highly attractive for the development of modern wireless communication systems such as $4^{\text {th }}$ Generation (4G) and Long Term Evolution (LTE) systems. So far, Orthogonal Frequency Division Multiplexing (OFDM) is the most popular and highly recommended modulation technique for Dynamic spectrum access (DSA) and Cognitive Radio (CR) applications. Despite their numerous advantages, OFDM system suffers from few drawbacks. Filter bank Multicarrier (FBMC), another modulation technique evolved from OFDM which overcomes these drawbacks is examined. FBMC applies filter banks and omits the severe out of band leakage of OFDM. This paper presents the comparative performance analysis of conventional OFDM system and FBMC system in terms of Signal to noise ratio (SNR), Bit error rate (BER), Packet delivery ratio(PDR) and number of packets dropped with the help of computer simulations performed using NETWORK SIMULATOR NS-2.34.
\end{abstract}

\section{Keywords}

OFDM, FBMC, DSA, OFDM-OQAM, NS2, MAC

\section{INTRODUCTION}

The growth of internet and the increasing demand of high data rate users have given Dynamic spectrum access networks a widespread attention in the recent years. In DSA networks, multiple nodes compete to utilize a shared frequency spectrum. OFDM with cyclic prefix (CP-OFDM), which uses an orthogonal set of subcarriers is by far the most popular case of multicarrier systems that has been proposed for the purpose of sharing the different subsets of these subcarriers among nodes [1],[2] deployed for DSA. However, the tight timing and synchronization requirement in OFDM is difficult to achieve in practice when the nodes belong to different administrative entities. Therefore, mutual interference among the signals of different users are resulted in case of an lack of synchronization. Moreover, the $\mathrm{CP}$ employed is purely redundant in terms of information and considerably reduces the bandwidth efficiency. The limitations of OFDM were overcome by a multicarrier communication system known as
FBMC first proposed by Saltzberg [3] which provides a better spectral shaping of subcarriers than OFDM systems. This is accomplished by the careful designing of the prototype filter, which simplifies equalization in the absence of $\mathrm{CP}$ and also promises a more efficient spectral utilization by minimizing interference across subcarriers [4]. By employing Offset Quadrature Amplitude Modulation (OQAM), the full capacity of the transmission bandwidth can be achieved in FBMC systems.

Using NETWORK SIMULATOR (NS-2.34), the most commonly used simulator for networking and wireless studies, both the modulation techniques i.e, FBMC and OFDM are simulated. Through extensive simulations, performance of both the systems are analyzed and compared in terms of SNR, BER, PDR and number of packets dropped.

\section{FBMC Vs OFDM}

FBMC is an advancement of OFDM. The modulators of OFDM and FBMC are shown in Fig.1. The only fundamental change is the replacement of the OFDM with a multicarrier system based on filter banks, wherein the IFFT plus CPin is replaced by the synthesis filter bank (SFB) while FFT plus CPout is replaced by the analysis filter bank (AFB).The basic principles of OFDM and FBMC are explained beow:

OFDM:

In OFDM system, the frequency spectrum of the subcarriers are overlapped with minimum frequency spacing and the orthogonality is achieved between the different subcarriers. . In fig.1, the input stream is split into parallel data streams using the serial to parallel (S/P) converter, which are then passed into an inverse fast Fourier transformation (IFFT) block to generate time sequence of the streams. Subsequently, by adding a cyclic prefix (CP), the OFDM symbol time sequences are extended. The CP is a copy of the last part of the symbol that is added in the beginning of the sequence and should be larger than the network delay spread inorder to mitigate the intersymbol interference (ISI) generated by the arrival of different OFDM symbols with different delay. The resulting digital signal is converted into analog form and transmitted over the channel. 
OFDM:

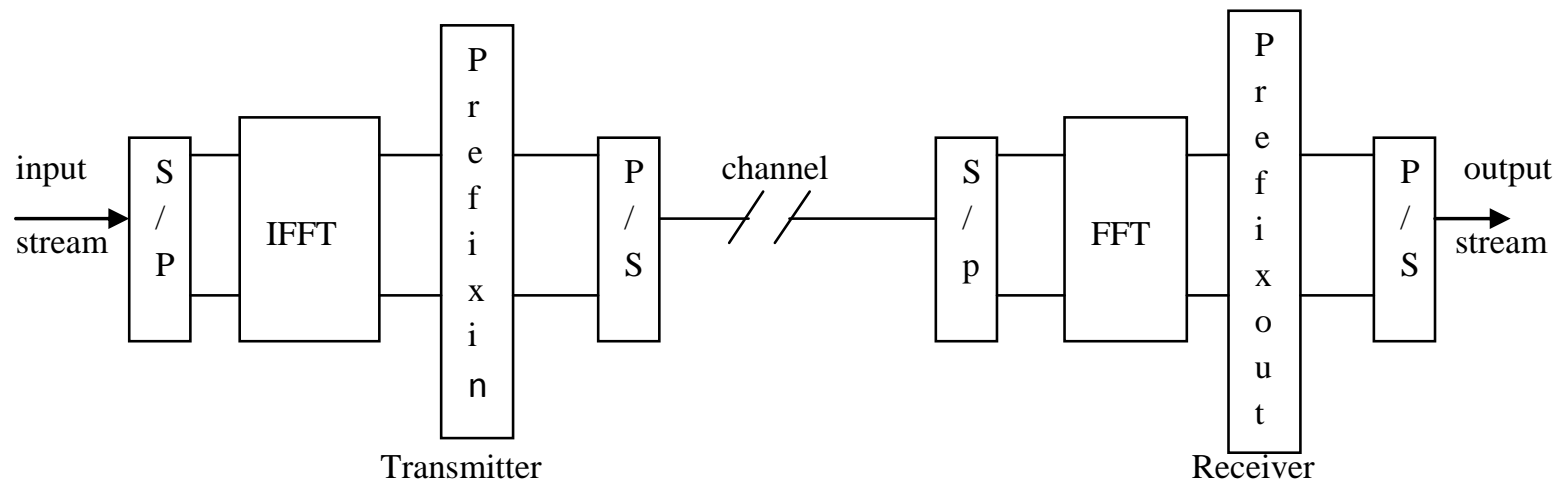

FBMC:

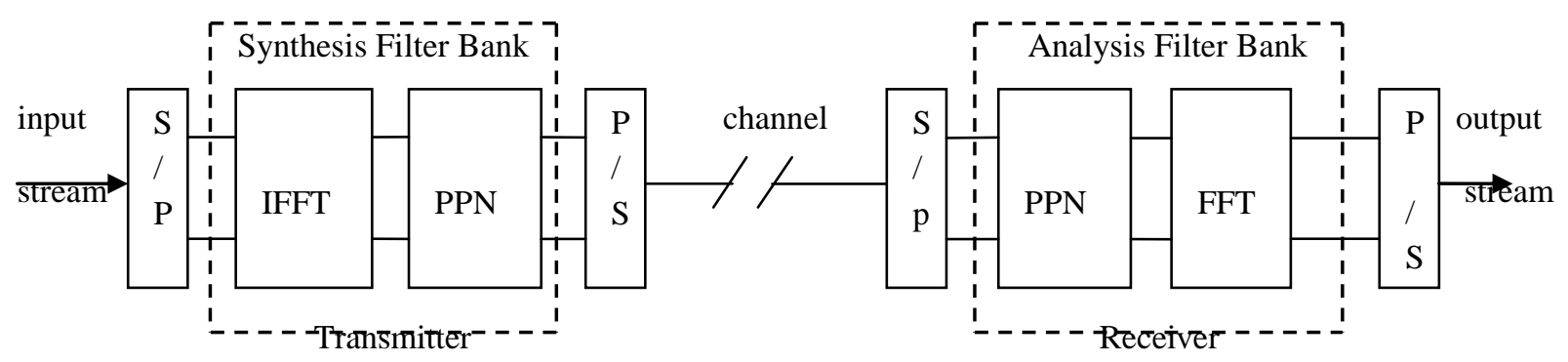

Fig 1: Modulators of OFDM and FBMC[5]

Table 1: Major differences between OFDM and FBMC systems

\begin{tabular}{|c|c|c|}
\hline Property & OFDM & FBMC \\
\hline CP Extension & $\begin{array}{c}\mathrm{CP} \text { extension required and therefore reduces } \\
\text { Bandwidth (BW) effeciency }\end{array}$ & Not required and hence conserves BW \\
\hline Sidelobes & Large sidelobes & Low sidelobes \\
\hline Synchronization & $\begin{array}{l}\text { For correct detection, multiple access } \\
\text { interference (MAI) cancellation should be } \\
\text { performed at the receiver }\end{array}$ & $\begin{array}{l}\text { MAI is suppressed due to the excellent } \\
\text { frequency localization of the subcarriers }\end{array}$ \\
\hline Doppler effect & $\begin{array}{l}\text { Highly sensitive to the carrier frequency } \\
\text { offset }\end{array}$ & $\begin{array}{l}\text { Less sensitive and hence performs } \\
\text { significantly with the increase of the user } \\
\text { mobility }\end{array}$ \\
\hline MIMO Systems & $\begin{array}{c}\text { High flexibility while adopting MIMO } \\
\text { techniques. }\end{array}$ & Limited flexibility \\
\hline $\begin{array}{l}\text { Spectrum } \\
\text { sensing }\end{array}$ & $\begin{array}{l}\text { Degraded spectrum sensing performance due } \\
\text { to the spectral leakage in OFDM signals }\end{array}$ & High spectrum sensing resolution \\
\hline $\begin{array}{l}\text { Computational } \\
\text { Complexity }\end{array}$ & Less complex & More complex \\
\hline
\end{tabular}

At the receiver, the signal is reconverted into digital form and the fast Fourier transform (FFT) is performed in the received streams after removing the CP. Lastly, the parallel streams are gathered into a single stream as the original transmitted one. Some of the drawbacks of OFDM are listed below:

$>$ Reduced spectral efficiency due to the CP employed

$>$ High spectral leakage due to the rectangular windowing
$>$ Interference between the unsysnchronized signal in the adjacent bands

FBMC:

FBMC overcomes the shortcomings of OFDM by adding generalized pulse shaping filters which produces a well localized subchannel in both time and frequency domain. Accordingly, FBMC systems have more spectral containment signals and provide more effective use of the radio resources where no $\mathrm{CP}$ is needed. 
Filterbanks can be defined as an array of $\mathrm{N}$ filters that processes $\mathrm{N}$ input signals to produce $\mathrm{N}$ outputs. If the inputs of these $\mathrm{N}$ filters are connected together, the system in analogous manner can be considered as an analyzer to the input signal based on each filter characteristics. Hence, this type of filter bank is called analysis filter bank (AFB). While on the other hand, by adding the outputs of the filter array, a new signal is synthesized and hence this type of filter bank is called synthesis filter bank (SFB)[6] . The synthesis-analysis configuration is called transmulitplexer and is applied in the MC communication systems[7].

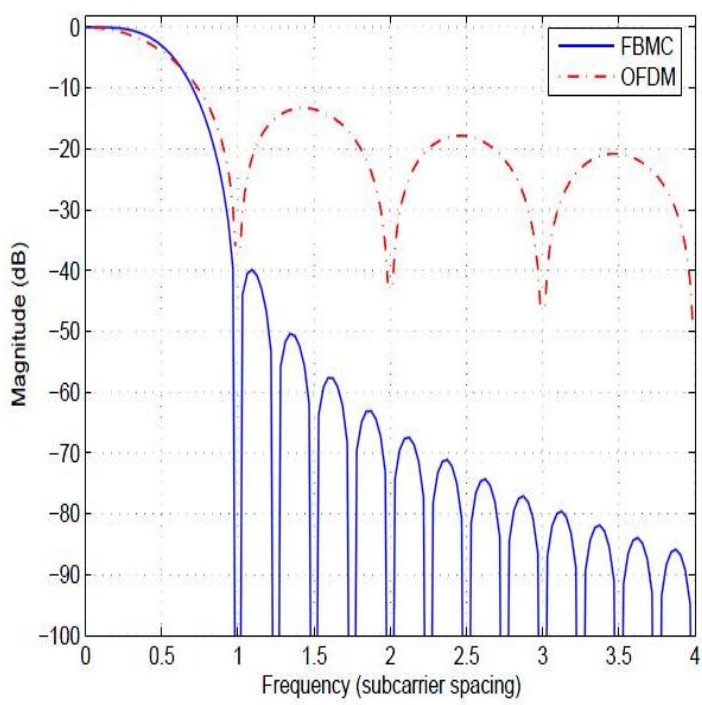

Fig 2: Frequency response of OFDM and FBMC [8].

The frequency response of OFDM and FBMC shown in Fig.2 reveals the impact of the transmission of data due to this exchange. Here, OFDM exhibits strong sidelobes due to the rectangular windowing, while on the other hand energy is concentrated within the frequency range of a single subcarrier in case of FBMC. Table 1 shows the major differences between OFDM and FBMC systems.

\section{SYSTEM MODEL}

The model is based on the operation of the PHY and MAC layer. The channel model and the different process inside them are assumed according to the IEEE 802.11 standard. We assumed the channel to be the default wireless channel class developed in NS2. The PHY entities are inserted in the list with the appropriate command in the TCL environment. The model implementation in NS2 has the basic link budget parameters for Wireless LAN technology stored in a header in the transmitted packet. For the node configuration, we assumed the two ray propagation model. For efficient handling of the data traffic, the widely used TCP protocol is used. Here, if a particular node is congested then the network coordinator identifies the congested node by applying its algorithm and delivers the packet to any neighbouring node which is less congested.

The process to determine if a packet is received correctly comprises of the following steps:

1. Estimation of the signal to noise ratio (SNRrx) at the receiver, based on the power received and the noise figure of the receptor.

2. Calculation of the bit error rate (BER) of the packet based on the SNRrx.

\section{SIMULATION SETUP AND RESULTS}

The NETWORK SIMULATOR NS-2.34 is used for the analysis. A snapshot of the simulation topology is shown in Fig.3.

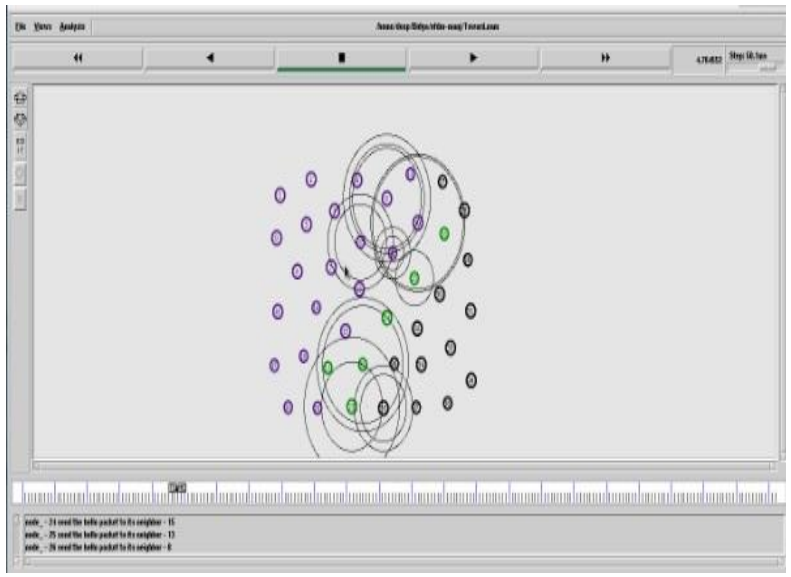

Fig 3: Snapshot of the NAM simulator

Here, the simulation scenarios are defined in the TCL environment to make use of the available entities with their corresponding parameters. For each of the entities, we had to define the proper TCL command to build and configure them. This enables mobile nodes to communicate among each other through wireless network interfaces, including the ability to simulate multihop wireless networks. We utilize 802.11 OFDM radio parameters at $5 \mathrm{GHZ}$ for packet data transfer among the $40(0-39)$ nodes deployed. The default wireless channel considered is of 20MHZ bandwidth. PHY data rates are upto $54 \mathrm{Mbps}$ maximum and transmits power varies from $0-20 \mathrm{dBm}$. Other physical layer parameters like path loss, fading, interference and noise computations are usually not taken into account in wireless simulations inspite of their important effects in simulation results [9]. 


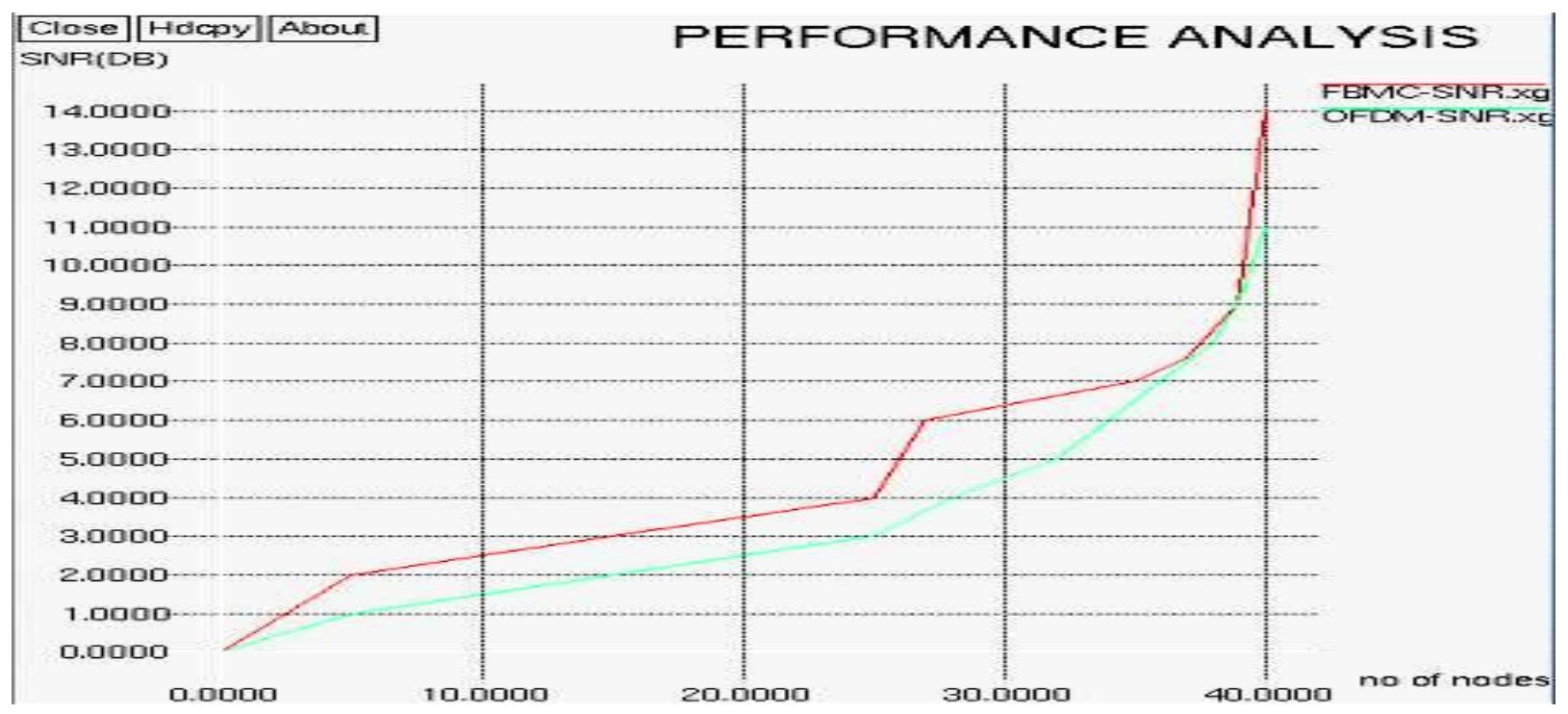

Fig 4: Comparison of Signal to Noise Ratio (SNR)

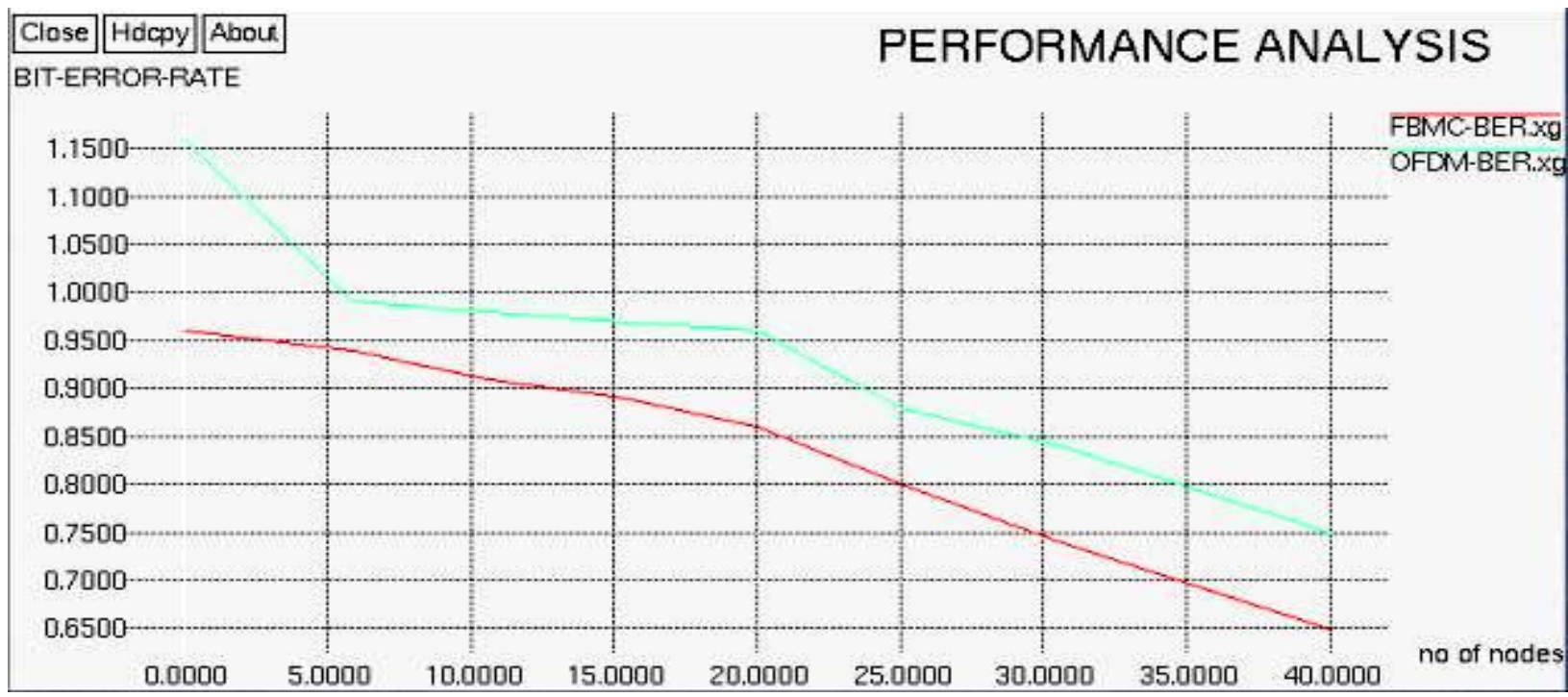

Fig 5: Comparison of bit error rate (BER)

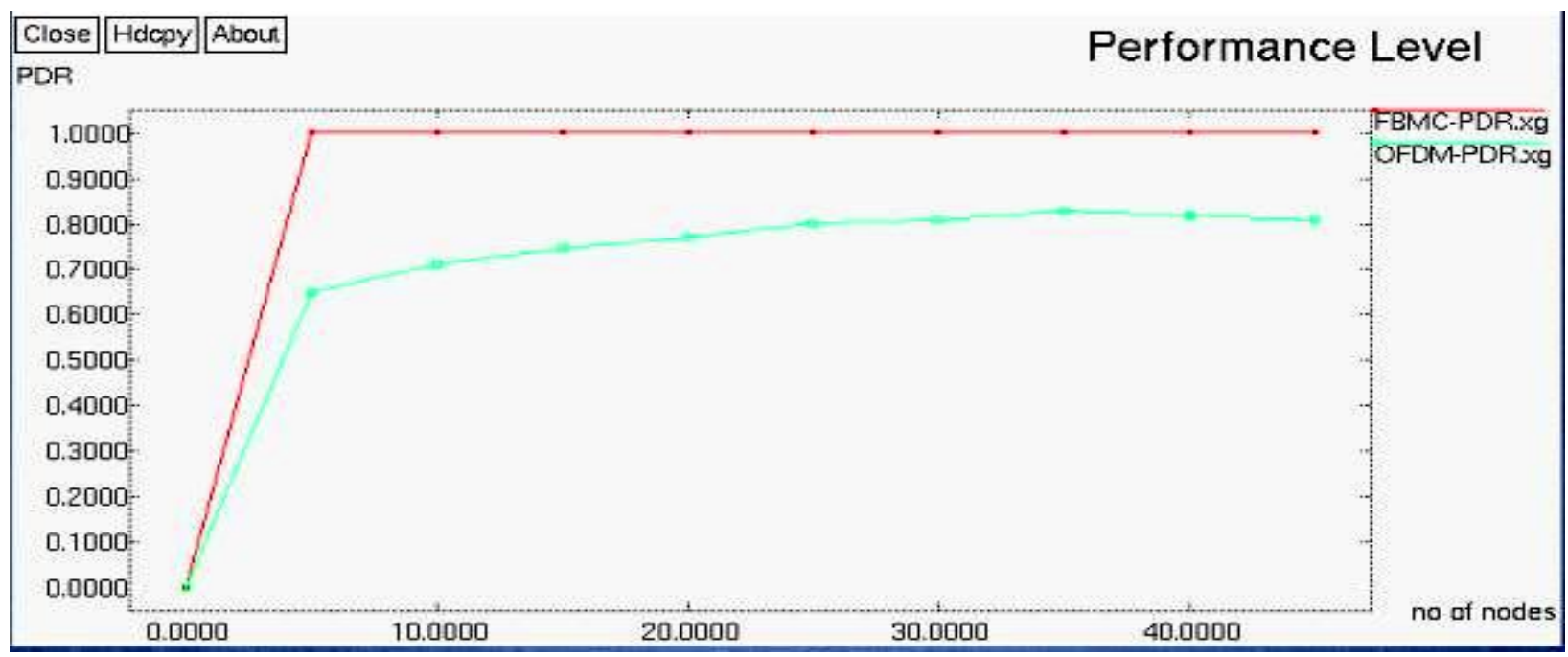

Fig 6: Comparison of Packet delivery ratio(PDR) 


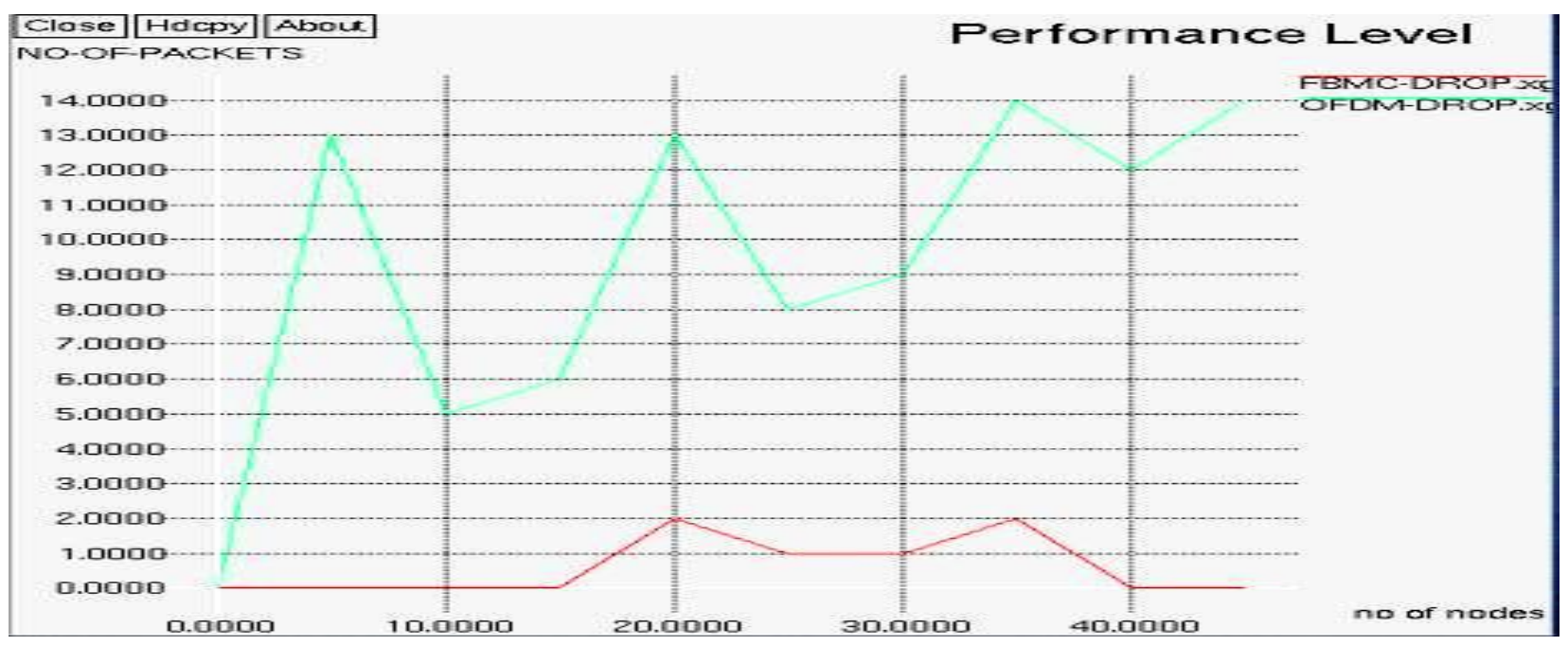

Fig 7: Comparison of dropped packets

\begin{abstract}
Also, the propagation model [10] is assumed to be the two ray propagation mode for the simulation. Further details of this simulation environment are available in [10]. Here, the default NS2 physical layer modulation comprising of OFDM-QAM is modified to the FBMC (OFDM-OQAM) system.
\end{abstract}

Fig 4 shows the comparison of signal to noise ratio of both the systems with respect to number of nodes. FBMC gives better SNR performance than the OFDM system. BER of OFDM is greater than FBMC and is shown in Fig 5.

Packet delivery ratio (PDR) may be defined as the ratio of number of delivered packets to the total number of packets transmitted. FBMC has higher PDR than OFDM and is shown in Fig 6. Fig 7 shows the comparison of dropped packets for each system. The dropped packets are the ones which have not reached the destination, even though a link is being established between the communication nodes. FBMC performs better than OFDM.

\section{CONCLUSION}

In this paper, the performance comparison between the most popular multicarrier modulation technique OFDM and a lesser known technique FBMC is performed in terms of Throughput, SNR, BER, packet delivery ratio and dropped packets. Simulation results show that FBMC gives overall performance improvement compared to conventional OFDM for all the parameters considered, proving FBMC as an ideal candidate for future development in wireless communications.

\section{REFERENCES}

[1] T. A. Weiss and F. K. Jondral, "Spectrum Pooling: An innovative strategy for the enhancement of spectrum efficiency," IEEE Communication Mag., vol. 42, no. 3, pp. S8-S14, Mar. 2004.

[2] D.J.Schafer , "Wide area adaptive spectrum applications," in Proc. IEEE MILCOM, 2001, vol. 1, pp. $1-5$.

[3] B.Saltzberg, "Performance of an efficient parallel data transmission system," IEEE Trans. Com. Tech, vol. 15, COM-6, pp.805-811, Dec. 1967.

[4] B. Farhang Boroujeny, “ A square root Nyquist (M) filter design for digital communication systems," IEEE Trans. Signal Process.,vol. 56.no.5,pp. 2127-2132, May 2008.

[5] Frank Schaich, "Filterbank based multicarrier transmission (FBMC)-evolving OFDM," Bell Labs Alcatel-Lucent, 2010.

[6] T.Hidalgo Stitz, "Filterbank techniques for the physical layer in wireless communications," Tampere University of Technology publications:919,2010.

[7] PP Vaidyanathan, "Multirate systems and filterbanks.1993".

[8] "PHYDYAS- Physical layer for dynamic spectrum access and cognitive radio," Project website: www.ictphydyas.org.

[9] M. Takai, J.Martin, and R.Bargodia, "Effects of Wireless Physical Layer modeling in Mobile Ad Hoc Networks," In Processing of MobiHoc 2001.

[10] G. Holland, N. Vaidya and P.Bahl, "A Rate Adaptive MAC Protocol for Multi-Hop Wireless Networks," MOBICOM, Rome, July 2001. 